\title{
Unexplained vascular pulmonary nodule
}

\author{
Fabio Davoli', Chiara Baldovini', Guido Caroli', Franco Stella ${ }^{3}$, Maria Teresa Minguzzi ${ }^{4}$, Giulio Rossi ${ }^{2}$ \\ 'Department of Thoracic Surgery, S. Maria delle Croci Teaching Hospital of Ravenna, Ravenna, Italy \\ ${ }^{2}$ Unit of Pathology, S. Maria delle Croci Hospital of Ravenna, Ravenna, Italy \\ ${ }^{3}$ University of Bologna, Department of Thoracic Surgery, Azienda USL Romagna, Morgagni Hospital, Forlì, Italy \\ ${ }^{4}$ Department of Radiology, Ausl della Romagna, S. Maria delle Croci Hospital, Ravenna, Italy
}

Incidental pulmonary nodules are commonly encountered in computed tomography (CT) imaging. The Fleischner Society Guidelines [1] estimate the risk of malignancy and give follow-up recommendations based on several factors such as size, attenuation, morphology, location, and time-dependent changes. Regardless, histologic examination is often required to achieve a correct diagnosis.

A 75-year-old Caucasian woman who was a non-smoker was referred to the Thoracic Surgery Department of our hospital for a slow-growing pulmonary nodule. Medical history and physical examination were unremarkable, and routine laboratory tests were normal. In 2018, she underwent a contrast-enhanced chest CT revealing a sub-centimetric nodule of the right lower lobe. One year later, a follow-up CT demonstrated an increase in volume $\left(169 \mathrm{~mm}^{3}\right.$ versus $86 \mathrm{~mm}^{3}$ ) (Figure 1), while a PET scan was negative. The differential diagnosis was between a benign lesion (hamartoma) or a low-grade neoplasm (tumorlet/carcinoid). Following the patient's choice and after multidisciplinary discussion, she underwent a video-assisted thoracoscopic (VATS) wedge resection. Since the nodule was not easily visible, a digital localization was performed which prevented conversion to thoracotomy. The postoperative course was characterized by hyperkalemia which was successfully treated by intravenous fluid administration. After discharge, the follow-up was uneventful and the patient is currently well.

The histologic examination revealed a nodular, well-defined proliferation originating from the wall of a peribronchiolar arteriole and protruding into the vascular lumen. The lesion was composed of bland-looking (absent cellular atypia, mitoses, and necrosis) cells, positive for smooth muscle actin and desmin in immunohistochemistry. A final diagnosis of vascular leiomyoma was established (Figure 2).

Muscular lesions of the lung encompass a limited number of entities including intimal/pulmonary artery sarcoma, leiomyosarcoma, benign metastasizing leiomyoma, leiomyomatous hamartoma, and naïve nodular smooth-muscle hyperplasia.

Pulmonary leiomyomas are extremely rare and significantly more frequent in females. Since they are often associated with a history of hysterectomy and/or uterine nodules, the great majority have been subsequently reconsidered as benign metastasizing leiomyomas [2]. However, the medical history of our patient did not include previous uterine nodules and imaging studies failed to reveal alterations of the gynecological tract. Therefore, such a diagnosis was excluded.

A case of pulmonary vascular leiomyoma was previously reported by Terada [3]. However, we believe that a diagnosis of nodular smooth-muscle hyperplasia would have been more appropriate in those circumstances since relevant images revealed a striking bronchiolocentric irregular growth of smooth muscle cells in close proximity to a regular arteriole [3]. The current case is unique in that the smooth muscle cell proliferation was limited to the arteriolar wall. Moreover, CT showed that the nodule was located along a small arteriole, which is in keeping with the histological features. The absence of similar observations in the literature led us to make an "arbitrary" diagnosis of vascular leiomyoma. Even if the pathogenesis remains unexplained, we chose this term to underline that it was a benign process originating from the smooth muscle component of the vascular wall. Overall, the description of this case might lead to a consideration of widening the spectrum of solitary nodules of the lung to include those likely arising from vascular structures of secondary lobules.

Address for correspondence: Fabio Davoli, Department of Thoracic Surgery, S. Maria delle Croci Teaching Hospital of Ravenna, Ravenna, Italy; e-mail: fabio.davoli78@gmail.com

DOI: 10.5603/ARM.a2020.0166

Received: 11.08.2020

Copyright (C) 2020 PTChP

ISSN 2451-4934

Conflict of interest: None declared 


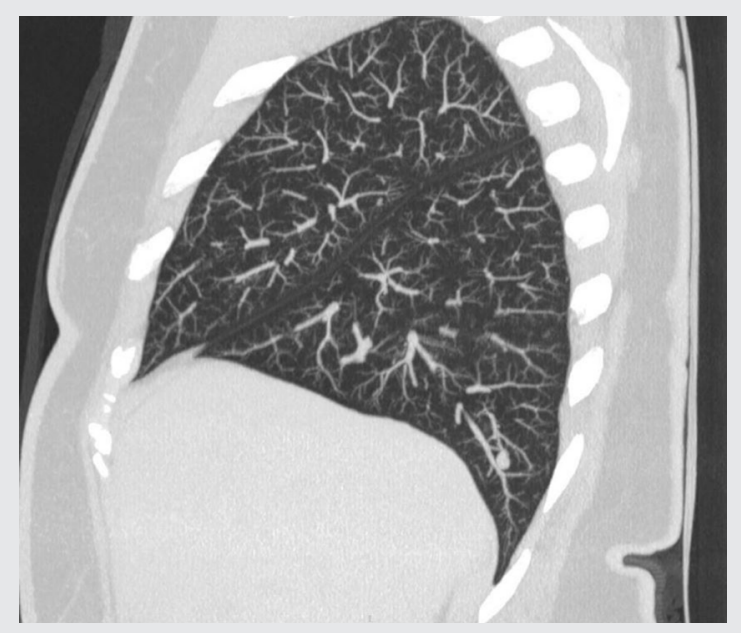

Figure 1. Chest computed tomography scan showing an unusual, rounded solid nodule involving a bronchiolar artery of the lateral segment of the right lower lobe

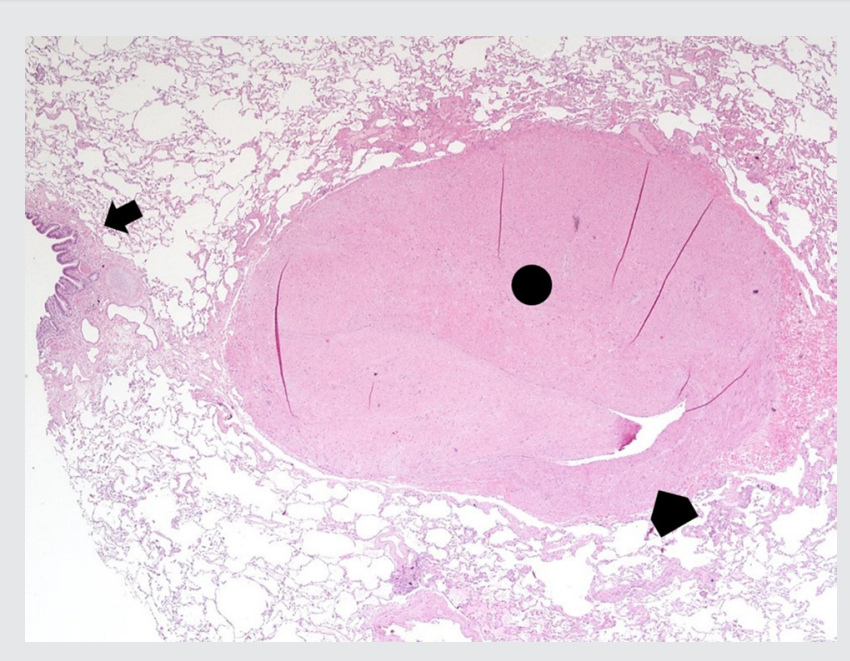

Figure 2. Histology confirmed a well-defined nodular growth of the smooth-muscle cell component (circle) of the arteriolar wall (arrowhead) in a bronchiolar bundle (arrow)

\section{References:}

1. MacMahon H, Naidich DP, Goo JMo, et al. Guidelines for Management of Incidental Pulmonary Nodules Detected on CT Images: From the Fleischner Society 2017. Radiology. 2017; 284(1): 228-243, doi: 10.1148/radiol.2017161659, indexed in Pubmed: 28240562.

2. Gal AA, Brooks JS, Pietra GG. Leiomyomatous neoplasms of the lung: a clinical, histologic, and immunohistochemical study. Mod Pathol. 1989; 2(3): 209-216, indexed in Pubmed: 2474816.

3. Terada T. Vascular leiomyoma of the lung arising from pulmonary artery. Int J Clin Exp Pathol. 2013; 6(1): 97-99, indexed in Pubmed: 23236548 . 